\title{
Long Noncoding RNA GAS5: A New Factor Involved in Bone Diseases
}

\author{
Zimo Zhou ${ }^{1}$, Jiahui Chen ${ }^{2}$, Ying Huang ${ }^{2}$, Da Liu ${ }^{1 *}$, Senxiang Chen ${ }^{1}$ and Sen Qin ${ }^{1}$ \\ ${ }^{1}$ Department of Orthopedics, Shengjing Hospital of China Medical University, Shenyang, China, ${ }^{2}$ Department of Ultrasound, \\ Shengjing Hospital of China Medical University, Shenyang, China
}

OPEN ACCESS

Edited by:

Jiake $X u$,

University of Western Australia, Australia

Reviewed by:

Chris Jones,

Brighton and Sussex Medical School, United Kingdom

Sean Shishu Huang,

Sichuan University, China

${ }^{*}$ Correspondence:

Da Liu

spinecmu@163.com

Specialty section:

This article was submitted to Molecular and Cellular Pathology, a section of the journal Frontiers in Cell and Developmental

Biology

Received: 02 November 2021 Accepted: 21 December 2021

Published: 26 January 2022

Citation:

Zhou Z, Chen J, Huang Y, Liu D, Chen $S$ and Qin $S$ (2022) Long Noncoding RNA GAS5: A New Factor Involved in Bone Diseases.

Front. Cell Dev. Biol. 9:807419. doi: 10.3389/fcell.2021.807419
Long noncoding RNAs (IncRNAs), as an important type of RNA encoded in the human transcriptome, have shown to regulate different genomic processes in human cells, altering cell type and function. These factors are associated with carcinogenesis, cancer metastasis, bone diseases, and immune system diseases, among other pathologies. Although many IncRNAs are involved in various diseases, the molecular mechanisms through which IncRNAs contribute to regulation of disease are still unclear. The IncRNA growth arrest-specific 5 (GAS5) is a key player that we initially found to be associated with regulating cell growth, differentiation, and development. Further work has shown that GAS5 is involved in the occurrence and prognosis of bone diseases, such as osteoporosis, osteosarcoma, and postosteoporotic fracture. In this review, we discuss recent progress on the roles of GAS5 in bone diseases to establish novel targets for the treatment of bone diseases.

Keywords: long noncoding RNA, Gas5, bone disease, osteogenic differentiation, stem cell

\section{INTRODUCTION}

As functional RNA molecules, noncoding RNAs (ncRNAs) include four broad categories: (1) microRNAs (miRNAs), (2) long ncRNAs (lncRNAs), (3) circular RNAs, and (4) pseudogenes. ncRNAs were previous thought to lack open reading frames (ORFs) and protein-coding potential. However, more recent studies have shown that ncRNAs are involved in the regulation of gene expression at the transcriptional and post-transcriptional levels (Zhou et al., 2020). Specifically, ncRNAs have roles in epigenetic regulation, chromatin remolding, protein modification, and RNA degradation (Beermann et al., 2016).

LncRNAs are regulatory ncRNAs 200-100,000 nucleotides (nt) in length. These molecules have been shown to play key roles in the occurrence and prognosis of diseases. As polyadenylated byproducts transcribed by RNA polymerase II, IncRNAs were initially thought to be a type of transcriptional noise without any real functions (Peng et al., 2018). However, advances in RNAbinding protein immunoprecipitation (Fuentes-Iglesias et al., 2020), RNA pull-down assays, and other sequencing technologies (Takahashi et al., 2012; Heather and Chain, 2016) have shown that lncRNAs regulate many complex processes in different diseases (Chen et al., 2020; Ghafouri-Fard et al., 2020; Zhang, Guo et al., 2020 Zhao, Pathak et al., 2020), including bone diseases such as osteoporosis, osteoarthritis (OA), ankylosing spondylitis (AS), osteosarcoma, and bone fracture (Figure 1). Furthermore, several studies have confirmed the involvement of lncRNAs in bone diseases (Li, Yun et al., 2020; Chatterjee et al., 2020; Hong et al., 2020; Kushlinskii et al., 2020). For example, Núnêz et al. (2000) showed that $H 19$ expression is downregulated in the myeloproliferative disease and that $H 19$ regulates insulin-like growth factor 2 in stem cells. Moreover, lncRNAs have also been shown to regulate the osteogenic differentiation of stem cells in bone diseases. 


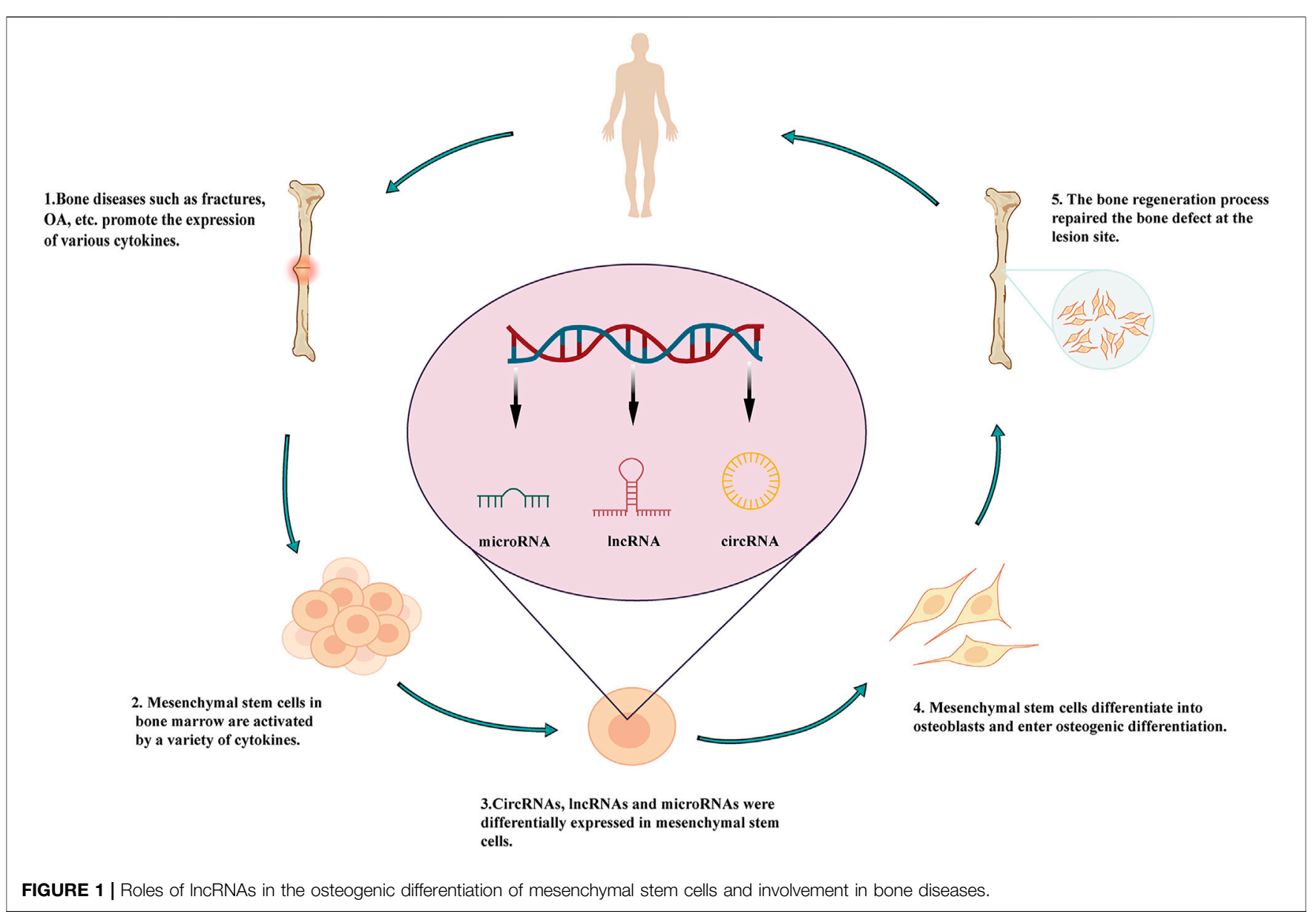

The lncRNA growth arrest-specific 5 (GAS5) was initially identified in NIH 3T3 cells using subtraction hybridization. In human cells, GAS5 was shown to be transcribed from a small ORF on chromosome 1q25.1 (Schneider et al., 1988; Isin et al., 2014). Moreover, GAS5 was subsequently shown to interact with the DNA binding domain of glucocorticoid receptors to suppress multiple anti-apoptotic genes, thereby enhancing the sensitivity of cells to apoptotic signals (Kino et al., 2010). Researchers are currently interested in evaluating the roles of GAS5 expression in bone diseases. For example, Visconti et al. (2020) found that GAS5 expression levels were significantly altered in the blood of patients with osteoporosis compared with that in healthy individuals. In another study, GAS5 was shown to be differentially expressed in patients with osteoporosis (Centofanti et al., 2020). However, few reports have evaluated the roles of GAS5 in bone diseases.

In this review, we discuss the expression of GAS5 in bone diseases and the mechanisms through which this lncRNA may contribute to the pathological state. Furthermore, we discuss problems with studies of GAS5 function in order to facilitate further research of this important lncRNA in bone diseases.

\section{REGULATORY MECHANISMS OF GAS5}

The GAS5 gene was first described by Schneider et al. (1988). Subsequently, Coccia et al. (1992) showed that the gene encoding
GAS5 has a $5^{\prime}$-terminal oligopyrimidine belonging to an upstream oligopyrimidine tract sequence. Since the transcription product of the GAS5 gene can accumulate in growth-arrested cells, the lncRNA is called growth arrestspecific 5 (GAS5). The gene is located on chromosome 1q25 and is $630 \mathrm{nt}$ in length, with 12 exons and a short ORF. When the exons are transcribed, the product can be spliced into two possible mature lncRNAs, GAS5a and GAS5b, through alternate splicing. Furthermore, during GAS5 transcription, there are many different patterns of alternate splicing. Notably, the activity of GAS5 may be associated with the introns, which encode small nucleolar RNAs (Shi et al., 2013).

In T cells, serum starvation and rapamycin treatment increase GAS5 expression via a mechanism involving the mammalian target of rapamycin pathway (Mourtada-Maarabouni et al., 2010). Kino et al. (2010) found that GAS5 increases sensitivity to apoptosis by inhibiting the anti-apoptotic activities of glucocorticoids. During this mechanism, GAS5, acting as a starvation- or growth arrestlinked riborepressor for the glucocorticoid receptor (GR), can bind to the DNA-binding domain, inhibiting the interaction between target DNA and its receptors.

In recent studies, GAS5 has been shown to be associated with tumors, functioning as a tumor suppressor, with roles in tumor occurrence, metastasis, necrosis, and prognosis (Zhao, Zheng et al., 2020). Moreover, in a screening of GAS5 expression in 
TABLE 1 | The roles of different microRNAs associated with GAS5.

\begin{tabular}{|c|c|c|c|c|}
\hline MicroRNA & Downstream target & Tumor or cell type & Effect & References \\
\hline miR-196a-5p & FOXO1/PI3K/AKT & & & Li Zhao et al. (2018) \\
\hline miR-221/222 & Unknown & & & Zong et al. (2019) \\
\hline$m i R-21$ & Unknown & & & Li et al. (2016) \\
\hline miR-205 & PTEN & Non-small cell lung cancer & Sponge & Dong et al. (2019) \\
\hline $\operatorname{miR}-135 b$ & Unknown & Cell lung & & Xue et al. (2017) \\
\hline $\operatorname{miR}-135 b$ & RECK & & & Yang et al. (2019) \\
\hline miRNA-34 & Different pathways & & & Toraih et al. (2018) \\
\hline$m i R-196 a-5 p$ & HOXA5 & Ovarian Cancer & Sponge & Zhao et al. (2018) \\
\hline
\end{tabular}

MSCs: mesenchymal stem cells; VSMCs: vascular smooth muscle cells; BMSCs: bone marrow mesenchymal stem cells; PDLSCs: periodontal ligament stem cells; ceRNA: competing endogenous RNA.

different breast cell lines, researchers showed that GAS5 transcriptional products with different exon/intron splicing combinations stimulate apoptosis through various cellular signaling pathways (Mourtada-Maarabouni et al., 2009).

\section{GAS5 as a Modulator of the Nucleoprotein Complex}

In placental mammals, chromatin silencing machinery, which is associated with the $\operatorname{lncRNA}$ /protein complex, has important biological roles, turning off the transcription of many genes on the inactive X chromosome (Penny et al., 1996). Biological techniques derived from these findings are widely used in various research fields. Importantly, ribosomes may bind to smORFs (small open reading frames) in one lncRNA without translating the ORF via ribosome "sponging", thereby inhibiting translation (Goustin et al., 2019).

\section{Regulation of Apoptosis by GAS5 as a Glucocorticoid Receptor Mimic}

GAS5 can fold into RNA secondary structures to compete with the GR for binding onto its target (Kino et al., 2010). Moreover, GAS5, as a competitive inhibitor of GR, can regulate cell apoptosis. In this mechanism, GR first binds to the GR ligand binding domain in the cytoplasm and then translocates into the nucleus to bind to specific GR DNA binding sequences to regulate the transcription of target genes. Studies are currently underway to use the GR element mimic to assess the lncRNA/protein binding domain in GAS5.

\section{Interaction of GAS5 Transcripts with miRNAs}

LncRNAs can serve as competing endogenous RNAs (ceRNAs), sponging the target miRNAs through homology similar to the miRNA/mRNA interaction, thereby regulating the expression and function of miRNAs (Liu et al., 2018). Several studies have shown that GAS5 can associate with miRNAs in many disease states. For example, GAS5 contributes to the development of breast cancer via a ceRNA-dependent mechanism. Like a sponge, GAS5 can directly associate with the binding site in
miRNA-23a, inactivating the miRNA-23a mimic and counteracting the negative effects of the miRNA-23a mimic on ATG3 to promote autophagy in breast cancer cells ( $\mathrm{Gu}$ et al., 2018b). In other studies of breast cancer, different miRNAs, including miR-221/222, miR-196a-5p, miR-378a-5p/SUFU, and $m i R-21$, have been shown to sponge with GAS5 (Li et al., 2016; Mei et al., 2017; Xue et al., 2017; Gu et al., 2018a; Li et al., 2018; Toraih et al., 2018; Zhao et al., 2018; Dong et al., 2019; Yang and Jiang, 2019; Zong et al., 2019; Wang, Ke et al., 2020; Zheng et al., 2020). These interactions have also been confirmed in other cancers (Table 1). However, the specific signaling pathways involved are still unclear, and further validation studies are needed to fully elucidate the roles of GAS5 in mediating miRNAs in various disease states.

\section{Other Possible Regulatory Mechanisms of GAS5}

GAS5 may also mediate disease states through various other mechanisms. In breast cancer, $\mathrm{Li}$ et al. (2019) found that GAS5 upregulation promotes the chemosensitivity and apoptosis of triple-negative breast cancer (TNBC) cells. Methylation of $\mathrm{CpG}$ islands in the promoter region of GAS5 was identified in TNBC tissues, indicating that aberrant methylation affects the biological activity of GAS5. Furthermore, upregulation of GAS5 via suppression of methylation was shown to accelerate apoptosis in TNBC cells.

In another study, GAS5 was found to be associated with the promoter element of the insulin receptor, altering its expression in patients with type 2 diabetes mellitus. Moreover, the stability of GAS5 is regulated by degradation rather than transcription. Indeed, limiting the degradation of GAS5 by blocking its interaction with upframeshift mutant (UPF1) increases GAS5 expression and glucose uptake in adipocytes from patients with diabetes (Goustin et al., 2019; Shi et al., 2019). These findings suggest that GAS5 may bind to genomic DNA to regulate various cellular processes.

YES-associated protein (YAP) activation plays key roles in cancer development by regulating target gene expression via formation of complexes with multiple transcription factors. Phosphorylation of a different locus in YAP, an important downstream locus of the Hippo pathway, can regulate nuclear 


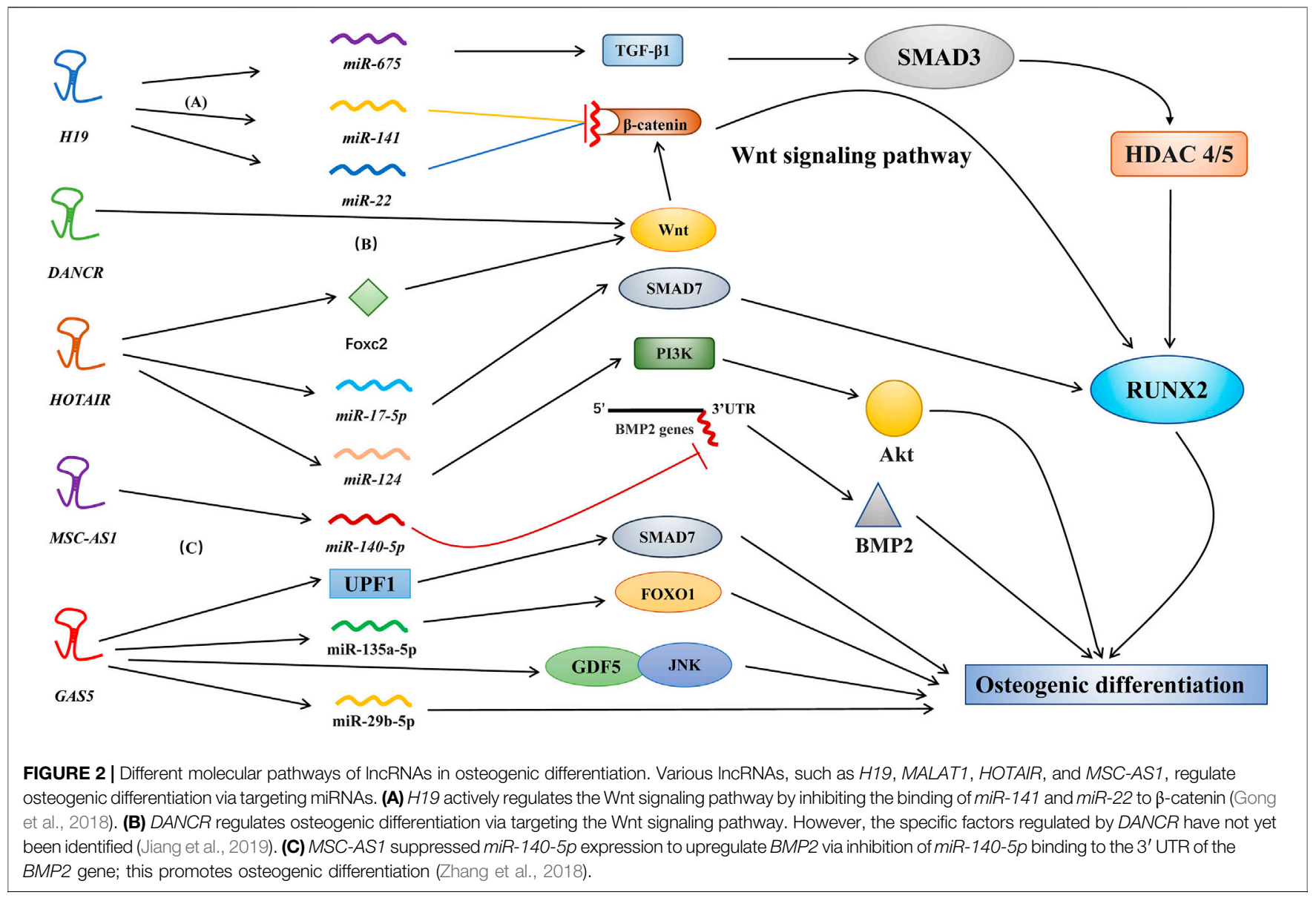

and cytoplasmic localization (Moon et al., 2017). The N6methyladenosine (m6A) modification is introduced by the m6A methyltransferase complex. Ni et al. (2019) showed that GAS5 can directly interact with the WW domain of YAP to promote the translocation of YAP from the nucleus to the cytoplasm and enhance the phosphorylation and ubiquitinmediated degradation of YAP. This mechanism results in decreased expression of the YAP-mediated transcription product of YTHDF3, which reversibly and selectively binds m6A-methylated GAS5 to trigger its decay, generating a negative feedback loop.

Accordingly, GAS5 may regulate different cellular metabolic pathways and processes to promote disease development. Further studies are needed to identify new GAS5-related treatment targets based on these mechanisms. Moreover, validation studies are needed to determine whether these mechanisms are truly involved in disease onset and progression.

\section{ROLES OF GAS5 IN THE OSTEOGENIC DIFFERENTIATION OF DIFFERENT TYPES OF STEM CELLS}

In several types of stem cells, osteogenic differentiation can promote bone regeneration and bone healing. Osteoblasts and osteoclasts are key cells involved in osteogenic differentiation and bone turnover. Osteoblasts are differentiated from bonederived stem cells, such as mesenchymal stem cells (MSCs), periodontal ligament stem cells (PDLSCs), and human dental pulp stem cells (Zhao, Tu et al., 2020; Kichenbrand et al., 2020; Tian et al., 2020). Various molecular pathways, including lncRNAs, regulate the complex osteogenic differentiation process. For example, in osteogenic differentiation, lncRNAs have been shown to modulate bone morphogenetic protein (BMP) (Zhang, Du et al., 2019), the WNT/ $\beta$-catenin/RUNX2 pathway (Shen et al., 2019; Zhou et al., 2019), and the transforming growth factor- $\beta$ (TGF- $\beta$ )/Smad3 pathway (Huang et al., 2015) (Figure 2) through three major mechanisms, including epigenetic modifications, miRNA sponges or precursors, and direct effects on molecular targets.

With advancements in genetic technologies, epigenetic modifications, including DNA methylation, histone modification, and RNA modulation have been analyzed in yeast, plants, and mammals. The important roles of lncRNAs in epigenetic, transcriptional, and post-transcriptional gene regulation, such as $\mathrm{X}$-chromosome inactivation, histone modification, imprinting, transcriptional interference, and nuclear and cytoplasmic trafficking, have been identified in all stages of osteogenic differentiation of stem cells (Martianov et al., 2007; Rinn et al., 2007; Skvortsova et al., 2018). 
TABLE 2 | Regulation and expression level of GAS5 in different MSCs.

\begin{tabular}{|c|c|c|c|c|c|}
\hline Molecular functions & $\begin{array}{l}\text { Expression level } \\
\text { of GAS5 } \\
\text { in osteoporosis }\end{array}$ & Targets & Cell model & Regulatory effect & References \\
\hline N/A & Overexpression & $\mathrm{N} / \mathrm{A}$ & Human blood & N/A & Goustin et al. (2019) \\
\hline N/A & Low expression & N/A & Human MSCs & N/A & Visconti et al. (2020) \\
\hline Nonsense-mediates mRNA decay & Low expression & UPF1 & Human MSCs & Promotion & Li, Yun et al. 2020 \\
\hline ceRNA & $\begin{array}{l}\text { Low expression } \\
\text { Low expression } \\
\text { Low expression }\end{array}$ & $\begin{array}{l}\text { miRNA-498/RUNX2 } \\
\text { miR-26b-5p/PTEN } \\
\text { miR-135a-5p/FOXO1 }\end{array}$ & $\begin{array}{l}\text { Human MSCs } \\
\text { Human VSMCs } \\
\text { Mouse BMSCs }\end{array}$ & $\begin{array}{l}\text { Inhibition } \\
\text { Inhibition } \\
\text { Promotion }\end{array}$ & $\begin{array}{l}\text { Feng et al.2019 } \\
\text { Leopold et al. (2015) } \\
\text { Wang et al. (2019) }\end{array}$ \\
\hline Phosphorylation & Low expression & GDF5 & Human PDLSCs & Promotion & Yang et al. (2020) \\
\hline
\end{tabular}

MSCs: mesenchymal stem cells; VSMCs: vascular smooth muscle cells; BMSCs: bone marrow mesenchymal stem cells; PDLSCs: periodontal ligament stem cells; ceRNA: competing endogenous RNA.

Furthermore, researchers have recently started evaluating lncRNA/miRNA sponges in osteogenic differentiation. For example, metastasis-associated lung adenocarcinoma transcript 1 (MALAT1) can sponge miR-30 in adipose-derived MSCs (Yi et al., 2019), miR-124 in MSCs (Zhang, Pathak. et al., 2020), and miR-204 in human aortic valve interstitial cells (Huang et al., 2015; Jiang et al., 2017; Wang et al., 2017; Wei et al., 2017; Xiao et al., 2017; Xiao et al., 2017; Wang, Zeng et al., 2018; Wang, Liu et al., 2018; Gao et al., 2018; Wu et al., 2018; Liu et al., 2019; Yang et al., 2019; Yi et al., 2019; Wang, Xiao et al., 2020; Zhang, Wang et al., 2020; Pan et al., 2020).

As an important regulatory factor, GAS5 is also involved in osteogenic differentiation. For example, GAS5 can regulate MSCs through various mechanisms and promote or inhibit the occurrence and development of bone diseases. Here, we summarize the potential mechanisms of action of GAS5 in various types of stem cells and discuss the regulatory effects involved in these mechanisms (Table 2).

\section{Human Mesenchymal Stem Cells}

MSCs are multipotent stem cells that can differentiate into a broad range of cell types, including osteoblasts, adipocytes, chondrocytes, tendon cells, and myocytes. MSCs have important potential in bone regeneration and tissue repair in bone, adipose, cartilage, and muscle tissues (Yang et al., 2018; Feng et al., 2019). Bone marrow MSCs (BMSCs) and adipose tissue-derived stromal stem cells are two types of MSCs that show high proliferative capacity and potential regenerative properties (Yang et al., 2018).

The regulation of different lncRNAs in MSCs has been widely reported. For example, the IncRNA H19 is involved in the osteogenic differentiation of MSCs, modulating various regulatory factors and pathways, such as the Wnt/ $\beta$-catenin pathway and the TGF- $\beta 1 / \mathrm{Smad} 3 /$ histone deacetylase (HDAC) signaling pathway (Huang et al., 2015; Zhou et al., 2019). However, the molecular mechanisms of GAS5 in MSCs are unclear. In one study, $\mathrm{Li}$ et al. (2020) found that GAS5 overexpression significantly enhanced alkaline phosphatase activity and promoted the osteogenic differentiation of human MSCs by interacting with UPF1 to degrade SMAD7 mRNA. These findings further established a novel pathway through which IncRNAs regulate nonsense-mediated mRNA decay, a highly conserved mechanism widely present in eukaryotes. In addition, GAS5 has been shown to modulate the miR-135a-5p/ FOXO1 pathway by functioning as a ceRNA (Wang et al., 2019). The GAS5/SMAD7 axis and GAS5/miR-135a-5p/FOXO1 axis in MSCs are both involved in the development and prognosis of osteoporosis and will be described in greater detail in Section 4.1.

\section{Human Periodontal Ligament Stem Cells}

Human periodontal ligament stem cells (hPDLSCs), as potential seed cells in bone engineering tissue, may contribute to alveolar bone regeneration (Jia et al., 2019). Knockdown of GAS5 inhibits the osteogenic differentiation of hPDLSCs. In contrast, overexpression of GAS5 promotes osteogenic differentiation. Yang et al. (2020) also found that GAS5 overexpression increases the level of growth differentiation factor 5 and accelerates the phosphorylation of JNK and p38 in hPDLSCs. However, the specific molecular mechanisms through which GAS5 functions in hPDLSCs remain unclear.

\section{Human Vascular Smooth Muscle Cells}

Vascular calcification plays important roles in the occurrence and development of cardiovascular disease and chronic kidney disease. This mechanism may be associated with the osteogenic differentiation of human vascular smooth muscle cells (hVSMCs) (Leopold, 2015). In one study, Chang et al. (2020) found that GAS5 was significantly downregulated in hVSMCs. Subsequent research showed that GAS5 overexpression positively regulates phosphatase and tensin homolog levels through $m i R-26 b$ $5 p$ sponging, thereby inhibiting osteogenic differentiation by inducing high levels of phosphorus in hVSMCs.

\section{ROLES OF GAS5 IN BONE DISEASES}

Bone diseases were previously thought to be disorders of the absorption and release of calcium and phosphorus. However, recent studies have demonstrated the involvement of bonederived cells and related molecules in bone diseases. Moreover, lncRNAs can also regulate the development of bone diseases. As a key factor in osteogenic differentiation, $\mathrm{H} 19$ has potential biological roles in bone diseases, such as osteoporosis ( $\mathrm{Li}$, 
Zhao et al., 2018; Xie et al., 2019), osteoarthritis (Steck et al., 2012; Chen et al., 2016), and fracture (Zhou, Yu et al., 2018; Li Yun et al., 2020). However, the roles of GAS5 in bone diseases are still unclear, and most studies of GAS5 in bone diseases have been limited to studies of osteoporosis and osteosarcoma.

\section{Osteoporosis}

Patients who have osteoporosis suffer from a higher risk of fractures. In older women in particular, the rate of fractures due to osteoporosis is greatly increased because of changes in estrogen levels (Väänänen and Härkönen, 1996). In the past, patients who had bone fractures often required surgery. In particular, patients with hip fractures often required hip replacements, resulting in poor quality of life, a dependent living situation, and an increased risk of death (Cummings and Melton, 2002). However, many recent clinical studies have focused on prevention of future fractures in patients with postmenopausal osteoporosis (Black and Rosen, 2016). Agerelated osteoporosis involves lack of new bone formation and the accumulation of fat in the bone marrow compartment, which can be associated with reduced osteoblast number and impaired differentiation of MSCs into adipocytes (Li, Xiao et al., 2018).

LncRNAs, as key factors regulating osteogenic differentiation, may have potential applications in the treatment of osteoporosis. For example, the novel lncRNA Bmncr modulates the age-related osteogenic niche (Li, Xiao et al., 2018). Indeed, Bmncr regulates the osteogenic niche of BMSCs by maintaining the expression of the extracellular matrix protein fibromodulin and the activation of the BMP2 pathway. Bmncr can also act as a scaffold to promote interaction of TAZ and ABL, thereby facilitating the assembly of the TAZ and RUNX2/PPARG transcriptional complex, promoting osteogenesis, and inhibiting adipogenesis.

GAS5 has also been shown to have key functions in osteoporosis. The GAS5 expression level is significantly downregulated in patients with osteoporosis (Centofanti et al., 2020). Moreover, Visconti et al. (2020) showed that GAS5 may have great value as a putative prognostic biomarker in patients with osteoporosis and osteoporosis-related fractures. In addition to its roles in the GAS5/ SMAD7 and GAS5/miR-135a-5p/FOXO1 axes, GAS5 also had other regulatory effects in osteoporosis. For example, quantitative real-time polymerase chain reaction showed that patients with osteoporosis exhibit high levels of $m i R-498$ and low levels of GAS5 and RUNX2. In further experiments, GAS5 was shown to significantly regulate RUNX5 expression via miR-498 (Feng et al., 2019). In other studies, GAS5 expression had altered in patients with osteoporosis (Centofanti et al., 2020; Visconti et al., 2020).

Overall, although many researchers are currently evaluating the mechanisms through which GAS5 mediates osteoporosis, information is still limited, and more robust studies are needed to confirm these findings. Furthermore, whether and how GAS5 regulates the same pathway through different miRNAs and the specific molecules that bind with GAS5 to modulate crosstalk among different pathways are still being investigated.

\section{Osteosarcoma}

Osteosarcoma is a common type of primary malignant tumor occurring primarily in children and adolescents. Osteosarcoma often arises in the long bones of the limbs, such as the femur, tibia, and humerus, near the metaphyseal growth plate (Zambo and Veselý, 2014). Surgery is still the major treatment strategy for patients with osteosarcoma; however, the survival rate of patients who undergo surgery alone is low. In recent decades, the combination of surgical treatment and chemotherapy has resulted in significantly increased survival rates (Kansara et al., 2014). However, metastasis, recurrence, and drug resistance seriously affect patient prognosis (Bielack et al., 2002; Ritter and Bielack, 2010). Therefore, analysis of the molecular mechanisms of osteosarcoma development may establish novel biomarkers for the treatment of this disease (Rossi et al., 2019; Czarnecka et al., 2020).

LncRNAs have also been shown to have important roles in osteosarcoma via regulation of multiple pathways in vivo and in vitro (Yan et al., 2018; Zhang et al., 2018; Fu et al., 2019; Zheng et al., 2019). Moreover, various $\operatorname{lncRNAs}$ alter tumorigenesis and metastasis by upregulation or downregulation of their targets. For example, the lncRNA SNHG12 promotes the development of osteosarcoma by upregulating Notch2 via sponging miR-195-5p (Zhou, Zhang et al., 2018).

Importantly, GAS5, which acts as a sponge for miR-221 (an miRNA that downregulates ARHI), exhibits antitumor effects and modulates the epithelial-mesenchymal transition by enhancing ARHI expression (Ye et al., 2017). As an alternate mechanism, HDAC1/2, which forms a heterodimer, can bind to the transcriptional complex composed of IRF1 and C-terminal binding protein 1 (CtBP1), called the CtBP1-HDAC1/2-IRF1 transcriptional complex; this complex targets the GAS5 promoter and suppresses GAS5 expression. In another study, downstream targets of GAS5, including tumorsuppressor genes (e.g., TP53, Bax, and Bim) as well as oncogenes (e.g., $T G F B, D D B 2$, and $R O S 1$ ), were identified (Zhang, Hu et al., 2019), further highlighting the roles of GAS5 in osteosarcoma.

\section{CONCLUSION}

In recent studies, researchers have shown that lncRNAs can be used as biomarkers for the prognosis, prevention, and treatment of various diseases, including bone-related diseases. Although the complex mechanisms through which IncRNAs contribute to diseases have not been fully elucidated, studies have demonstrated their potential applications in clinical practice. GAS5, a key regulatory molecule, can suppress cell growth to modulate the disease state. In normal cells, GAS5's expression is low; however, in tumor tissues and other diseased tissues, GAS5 is upregulated. With the development of biotechnologies, such as sequencing-based methods (Qian et al., 2019), researchers will be able to elucidate additional signaling pathways and targets. Moreover, additional work is still needed to confirm the findings reported in the current body of literature. For example, in vivo experiments are required to validate the roles of GAS5 demonstrated in basic cellular studies. In addition, many of the details of GAS5 activity and mechanisms are still unclear. Furthermore, whether and how GAS5 regulates relevant mechanisms in different bone-derived stem cells, whether and how GAS5 regulates the same pathway mediated by different miRNAs, which molecules harbor GAS5 binding sites to facilitate crosstalk among different pathways, and whether GAS5 has roles 
in other bone diseases, such as OA and AS have not yet been clarified. Therefore, intensive discussions are required to decide how to dissect the roles of GAS5 in particular systems, such as bone tissue. Finally, further studies are necessary to translate basic research into clinical practice.

\section{AUTHOR CONTRIBUTIONS}

ZZ designed the work and drafted the manuscript. JC, YH, SC, and SQ revised the manuscript. DL revised the manuscript and approved the work. All authors read and approved the manuscript.

\section{REFERENCES}

Beermann, J., Piccoli, M.-T., Viereck, J., and Thum, T. (2016). Non-coding RNAs in Development and Disease: Background, Mechanisms, and Therapeutic Approaches. Physiol. Rev. 96 (4), 1297-1325. doi:10.1152/physrev.00041.2015

Bielack, S. S., Kempf-Bielack, B., Delling, G., Exner, G. U., Flege, S., Helmke, K., et al. (2002). Prognostic Factors in High-Grade Osteosarcoma of the Extremities or Trunk: an Analysis of 1,702 Patients Treated on Neoadjuvant Cooperative Osteosarcoma Study Group Protocols. Jco 20 (3), 776-790. doi:10. $1200 /$ jco.2002.20.3.776

Black, D. M., and Rosen, C. J. (2016). Postmenopausal Osteoporosis. N. Engl. J. Med. 374 (3), 254-262. doi:10.1056/NEJMcp1513724

Centofanti, F., Santoro, M., Marini, M., Visconti, V. V., Rinaldi, A. M., Celi, M., et al. (2020). Identification of Aberrantly-Expressed Long Non-coding RNAs in Osteoblastic Cells from Osteoporotic Patients. Biomedicines 8 (3), 65. doi:10. 3390/biomedicines 8030065

Chang, Z., Yan, G., Zheng, J., and Liu, Z. (2020). The IncRNA GAS5 Inhibits the Osteogenic Differentiation and Calcification of Human Vascular Smooth Muscle Cells. Calcif Tissue Int. 107 (1), 86-95. doi:10.1007/s00223-02000696-1

Chatterjee, S., Bhattcharjee, D., Misra, S., Saha, A., Bhattacharyya, N. P., and Ghosh, A. (2020). Increase in MEG3, MALAT1, NEAT1 Significantly Predicts the Clinical Parameters in Patients with Rheumatoid Arthritis. Personalized Med. 17, 445-457. doi:10.2217/pme-2020-0009

Chen, S.-w., Wang, P.-y., Liu, Y.-c., Sun, L., Zhu, J., Zuo, S., et al. (2016). Effect of Long Noncoding RNA H19 Overexpression on Intestinal Barrier Function and its Potential Role in the Pathogenesis of Ulcerative Colitis. Inflamm. Bowel Dis. 22 (11), 2582-2592. doi:10.1097/mib.0000000000000932

Chen, Z., Huang, J., Feng, Y., Li, Z., and Jiang, Y. (2021). Profiling of Specific Long Non-coding RNA Signatures Identifies ST8SIA6-AS1 AS a Novel Target for Breast Cancer. J. Gene Med. 23 (2), e3286. doi:10.1002/jgm.3286

Coccia, E. M., Cicala, C., Charlesworth, A., Ciccarelli, C., Rossi, G. B., Philipson, L., et al. (1992). Regulation and Expression of a Growth Arrest-specific Gene (Gas5) during Growth, Differentiation, and Development. Mol. Cel Biol 12 (8), 3514-3521. doi:10.1128/mcb.12.8.351410.1128/mcb.12.8.3514-3521.1992

Cummings, S. R., and Melton, L. J. (2002). Epidemiology and Outcomes of Osteoporotic Fractures. The Lancet 359 (9319), 1761-1767. doi:10.1016/ s0140-6736(02)08657-9

Czarnecka, A. M., Synoradzki, K., Firlej, W., Bartnik, E., Sobczuk, P., Fiedorowicz, M., et al. (2020). Molecular Biology of Osteosarcoma. Cancers 12 (8), 2130. doi:10.3390/cancers 12082130

Dong, L., Li, G., Li, Y., and Zhu, Z. (2019). Upregulation of Long Noncoding RNA GAS5 Inhibits Lung Cancer Cell Proliferation and Metastasis via miR-205/PTEN Axis. Med. Sci. Monit. 25, 2311-2319. doi:10.12659/msm. 912581

Feng, J., Wang, J. X., and Li, C. H. (2019). LncRNA GAS5 Overexpression Alleviates the Development of Osteoporosis through Promoting Osteogenic Differentiation of MSCs via Targeting microRNA-498 to Regulate RUNX2. Eur. Rev. Med. Pharmacol. Sci. 23 (18), 7757-7765. doi:10.26355/ eurrev_201909_18985

\section{FUNDING}

This work was supported by grants from the National Natural Science Foundation of China (grant no. 81972156), the Natural Science Foundation of Liaoning Province (grant no. 2019-ZD0781), and the 345 Talent Project.

\section{ACKNOWLEDGMENTS}

We thank the laboratories affiliated with China Medical University for core support.

Fu, D., Lu, C., Qu, X., Li, P., Chen, K., Shan, L., et al. (2019). LncRNA TTN-AS1 Regulates Osteosarcoma Cell Apoptosis and Drug Resistance via the miR-1345p/MBTD1 axis. Aging 11 (19), 8374-8385. doi:10.18632/aging.102325

Fuentes-Iglesias, A., Garcia-Outeiral, V., Pardavila, J. A., Wang, J., Fidalgo, M., and Guallar, D. (2020). An Optimized Immunoprecipitation Protocol for Assessing Protein-RNA Interactions In Vitro. STAR Protoc. 1 (2), 100093. doi:10.1016/j. xpro.2020.100093

Gao, Y., Xiao, F., Wang, C., Wang, C., Cui, P., Zhang, X., et al. (2018). Long Noncoding RNA MALAT1 Promotes Osterix Expression to Regulate Osteogenic Differentiation by Targeting miRNA-143 in Human Bone Marrow-derived Mesenchymal Stem Cells. J. Cel. Biochem. 119 (8), 6986-6996. doi:10.1002/jcb.26907

Ghafouri-Fard, S., Eghtedarian, R., and Taheri, M. (2020). The Crucial Role of Non-coding RNAs in the Pathophysiology of Inflammatory Bowel Disease. Biomed. Pharmacother. 129, 110507. doi:10.1016/j.biopha.2020.110507

Gong, Y. Y., Peng, M. Y., Yin, D. Q., and Yang, Y. F. (2018). Long Non-coding RNA H19 Promotes the Osteogenic Differentiation of Rat Ectomesenchymal Stem Cells via Wnt/ $\beta$-Catenin Signaling Pathway. Eur. Rev. Med. Pharmacol. Sci. 22 (24), 8805-8813. doi:10.26355/eurrev_201812_16648

Goustin, A., Thepsuwan, P., Kosir, M., and Lipovich, L. (2019). The GrowthArrest-Specific (GAS)-5 Long Non-coding RNA: A Fascinating lncRNA Widely Expressed in Cancers. ncRNA 5 (3), 46. doi:10.3390/ncrna5030046

Gu, J., Wang, Y., Wang, X., Zhou, D., Shao, C., Zhou, M., et al. (2018a). RETRACTED: Downregulation of IncRNA GAS5 Confers Tamoxifen Resistance by Activating miR-222 in Breast Cancer. Cancer Lett. 434, 1-10. doi:10.1016/j.canlet.2018.06.039

Gu, J., Wang, Y., Wang, X., Zhou, D., Wang, X., Zhou, M., et al. (2018b). Effect of the LncRNA GAS5-MiR-23a-ATG3 Axis in Regulating Autophagy in Patients with Breast Cancer. Cell Physiol Biochem 48 (1), 194-207. doi:10.1159/ 000491718

Heather, J. M., and Chain, B. (2016). The Sequence of Sequencers: The History of Sequencing DNA. Genomics 107 (1), 1-8. doi:10.1016/j.ygeno.2015.11.003

Hong, J., Ye, F., Yu, B., Gao, J., Qi, F., and Wang, W. (2020). Identification of the Specific microRNAs and Competitive Endogenous RNA Mechanisms in Osteoporosis. J. Int. Med. Res. 48 (10), 030006052095472. doi:10.1177/ 0300060520954722

Huang, Y., Zheng, Y., Jia, L., and Li, W. (2015). Long Noncoding RNA H 19 Promotes Osteoblast Differentiation Via TGF - $\beta 1 / \mathrm{S}$ Mad3/HDAC Signaling Pathway by Deriving Mi R -675. Stem Cells 33 (12), 3481-3492. doi:10.1002/ stem. 2225

Isin, M., Ozgur, E., Cetin, G., Erten, N., Aktan, M., Gezer, U., et al. (2014). Investigation of Circulating lncRNAs in B-Cell Neoplasms. Clinica Chim. Acta 431, 255-259. doi:10.1016/j.cca.2014.02.010

Jia, L., Zhang, Y., Ji, Y., Xiong, Y., Zhang, W., Wen, Y., et al. (2019). YAP Balances the Osteogenic and Adipogenic Differentiation of hPDLSCs In Vitro Partly through the Wnt/ $\beta$-Catenin Signaling Pathway. Biochem. Biophysical Res. Commun. 518 (1), 154-160. doi:10.1016/j.bbrc.2019.08.024

Jiang, N., Wang, X., Xie, X., Liao, Y., Liu, N., Liu, J., et al. (2017). IncRNA DANCR Promotes Tumor Progression and Cancer Stemness Features in Osteosarcoma by Upregulating AXL via miR-33a-5p Inhibition. Cancer Lett. 405, 46-55. doi:10.1016/j.canlet.2017.06.009 
Jiang, S. Y., Miao, Y. X., Hirokazu, T., Zhu, S. Z., and Lu, J. S. (2019). Effects of IncRNA DANCR on Proliferation and Differentiation of Osteoblasts by Regulating the Wnt//-Catenin Pathway. Eur. Rev. Med. Pharmacol. Sci. 23 (13), 5558-5566. doi:10.26355/eurrev_201907_18289

Kansara, M., Teng, M. W., Smyth, M. J., and Thomas, D. M. (2014). Translational Biology of Osteosarcoma. Nat. Rev. Cancer 14 (11), 722-735. doi:10.1038/ nrc3838

Kichenbrand, C., Grossin, L., Menu, P., and Moby, V. (2020). Behaviour of Human Dental Pulp Stem Cell in High Glucose Condition: Impact on Proliferation and Osteogenic Differentiation. Arch. Oral Biol. 118, 104859. doi:10.1016/j. archoralbio.2020.104859

Kino, T., Hurt, D. E., Ichijo, T., Nader, N., and Chrousos, G. P. (2010). Noncoding RNA Gas5 Is a Growth Arrest- and Starvation-Associated Repressor of the Glucocorticoid Receptor. Sci. Signal. 3 (107), ra8. doi:10.1126/scisignal.2000568

Kushlinskii, N. E., Fridman, M. V., and Braga, E. A. (2020). Long Non-coding RNAs as Competitive Endogenous RNAs in Osteosarcoma. Mol. Biol. (Mosk) 54 (5), 776-801. doi:10.31857/S0026898420050055

Leopold, J. A. (2015). Vascular Calcification: Mechanisms of Vascular Smooth Muscle Cell Calcification. Trends Cardiovasc. Med. 25 (4), 267-274. doi:10. 1016/j.tcm.2014.10.021

Li, B., Zhao, J., Ma, J.-x., Li, G.-m., Zhang, Y., Xing, G.-s., et al. (2018a). Overexpression of DNMT1 Leads to Hypermethylation of H19 Promoter and Inhibition of Erk Signaling Pathway in Disuse Osteoporosis. Bone 111, 82-91. doi:10.1016/j.bone.2018.03.017

Li, C.-J., Xiao, Y., Yang, M., Su, T., Sun, X., Guo, Q., et al. (2018b). Long Noncoding RNA Bmncr Regulates Mesenchymal Stem Cell Fate during Skeletal Aging. J. Clin. Invest. 128 (12), 5251-5266. doi:10.1172/jci99044

Li, G., Yun, X., Ye, K., Zhao, H., An, J., Zhang, X., et al. (2020a). Long Non-coding RNA-H19 Stimulates Osteogenic Differentiation of Bone Marrow Mesenchymal Stem Cells via the microRNA-149/SDF-1 axis. J. Cel Mol Med 24 (9), 4944-4955. doi:10.1111/jcmm.15040

Li, J., Li, L., Yuan, H., Huang, X.-W., Xiang, T., and Dai, S. (2019). Up-regulated IncRNA GAS5 Promotes Chemosensitivity and Apoptosis of Triple-Negative Breast Cancer Cells. Cell Cycle 18 (16), 1965-1975. doi:10.1080/15384101.2019. 1635870

Li, M., Xie, Z., Li, J., Lin, J., Zheng, G., Liu, W., et al. (2020b). GAS5 Protects against Osteoporosis by Targeting UPF1/SMAD7 axis in Osteoblast Differentiation. Elife 9, e59079. doi:10.7554/eLife.59079

Li, S., Zhou, J., Wang, Z., Wang, P., Gao, X., and Wang, Y. (2018). Long Noncoding RNA GAS5 Suppresses Triple Negative Breast Cancer Progression through Inhibition of Proliferation and Invasion by Competitively Binding miR-196a5p. Biomed. Pharmacother. 104, 451-457. doi:10.1016/j.biopha.2018.05.056

Li, W., Zhai, L., Wang, H., Liu, C., Zhang, J., Chen, W., et al. (2016). Downregulation of LncRNA GAS5 Causes Trastuzumab Resistance in Breast Cancer. Oncotarget 7 (19), 27778-27786. doi:10.18632/oncotarget.8413

Liu, B., Wu, S., Ma, J., Yan, S., Xiao, Z., Wan, L., et al. (2018). IncRNA GAS5 Reverses EMT and Tumor Stem Cell-Mediated Gemcitabine Resistance and Metastasis by Targeting miR-221/SOCS3 in Pancreatic Cancer. Mol. Ther. Nucleic Acids 13, 472-482. doi:10.1016/j.omtn.2018.09.026

Liu, Y., Liu, C., Zhang, A., Yin, S., Wang, T., Wang, Y., et al. (2019). Downregulation of Long Non-coding RNA MEG3 Suppresses Osteogenic Differentiation of Periodontal Ligament Stem Cells (PDLSCs) through miR27a-3p/IGF1 axis in Periodontitis. Aging 11 (15), 5334-5350. doi:10.18632/ aging.102105

Martianov, I., Ramadass, A., Serra Barros, A., Chow, N., and Akoulitchev, A. (2007). Repression of the Human Dihydrofolate Reductase Gene by a Noncoding Interfering Transcript. Nature 445 (7128), 666-670. doi:10.1038/ nature 05519

Mei, Y., Si, J., Wang, Y., Huang, Z., Zhu, H., Feng, S., et al. (2017). Long Noncoding RNA GAS5 Suppresses Tumorigenesis by Inhibiting miR-23a Expression in Non-Small Cell Lung Cancer. Oncol. Res. 25 (6), 1027-1037. doi:10.3727/ 096504016x14822800040451

Moon, S., Kim, W., Kim, S., Kim, Y., Song, Y., Bilousov, O., et al. (2017). Phosphorylation by NLK Inhibits YAP -14-3-3-interactions and Induces its Nuclear Localization. EMBO Rep. 18 (1), 61-71. doi:10.15252/embr.201642683

Mourtada-Maarabouni, M., Hasan, A. M., Farzaneh, F., and Williams, G. T. (2010). Inhibition of Human T-Cell Proliferation by Mammalian Target of Rapamycin (mTOR) Antagonists Requires Noncoding RNA Growth-arrest-specific
Transcript 5 (GAS5). Mol. Pharmacol. 78 (1), 19-28. doi:10.1124/mol.110. 064055

Mourtada-Maarabouni, M., Pickard, M. R., Hedge, V. L., Farzaneh, F., and Williams, G. T. (2009). GAS5, a Non-protein-coding RNA, Controls Apoptosis and Is Downregulated in Breast Cancer. Oncogene 28 (2), 195-208. doi:10.1038/onc.2008.373

Ni, W., Yao, S., Zhou, Y., Liu, Y., Huang, P., Zhou, A., et al. (2019). Long Noncoding RNA GAS5 Inhibits Progression of Colorectal Cancer by Interacting with and Triggering YAP Phosphorylation and Degradation and Is Negatively Regulated by the m6A Reader YTHDF3. Mol. Cancer 18 (1), 143. doi:10.1186/s12943-019-1079-y

Núnêz, C., Bashein, A. M., Brunet, C. L., Hoyland, J. A., Freemont, A. J., Buckle, A. M., et al. (2000). Expression of the Imprinted Tumour-Suppressor Gene H19 Is Tightly Regulated during normal Haematopoiesis and Is Reduced in Haematopoietic Precursors of Patients with the Myeloproliferative Disease Polycythaemia Vera. J. Pathol. 190 (1), 61-68. doi:10.1002/(SICI)10969896(200001)190:1<61:AID-PATH502>3.0.CO;2-\#

Pan, Z., Wu, C., Li, Y., Li, H., An, Y., Wang, G., et al. (2020). LncRNA DANCR Silence Inhibits SOX5-Medicated Progression and Autophagy in Osteosarcoma via Regulating miR-216a-5p. Biomed. Pharmacother. 122, 109707. doi:10.1016/ j.biopha.2019.109707

Peng, S., Cao, L., He, S., Zhong, Y., Ma, H., Zhang, Y., et al. (2018). An Overview of Long Noncoding RNAs Involved in Bone Regeneration from Mesenchymal Stem Cells. Stem Cell Int. 2018, 1-11. doi:10.1155/2018/8273648

Penny, G. D., Kay, G. F., Sheardown, S. A., Rastan, S., and Brockdorff, N. (1996). Requirement for Xist in X Chromosome Inactivation. Nature 379 (6561), 131-137. doi:10.1038/379131a0

Qian, X., Zhao, J., Yeung, P. Y., Zhang, Q. C., and Kwok, C. K. (2019). Revealing IncRNA Structures and Interactions by Sequencing-Based Approaches. Trends Biochem. Sci. 44 (1), 33-52. doi:10.1016/j.tibs.2018.09.012

Rinn, J. L., Kertesz, M., Wang, J. K., Squazzo, S. L., Xu, X., Brugmann, S. A., et al. (2007). Functional Demarcation of Active and Silent Chromatin Domains in Human HOX Loci by Noncoding RNAs. Cell 129 (7), 1311-1323. doi:10.1016/j. cell.2007.05.022

Ritter, J., and Bielack, S. S. (2010). Osteosarcoma. Ann. Oncol. 21 (Suppl. 7), vii320-vii325. doi:10.1093/annonc/mdq276

Rossi, F., Tortora, C., Punzo, F., Bellini, G., Argenziano, M., Di Paola, A., et al. (2019). The Endocannabinoid/Endovanilloid System in Bone: From Osteoporosis to Osteosarcoma. Ijms 20 (8), 1919. doi:10.3390/ijms20081919

Schneider, C., King, R. M., and Philipson, L. (1988). Genes Specifically Expressed at Growth Arrest of Mammalian Cells. Cell 54 (6), 787-793. doi:10.1016/s00928674(88)91065-3

Shen, J. J., Zhang, C. H., Chen, Z. W., Wang, Z. X., Yang, D. C., Zhang, F. L., et al. (2019). LncRNA HOTAIR Inhibited Osteogenic Differentiation of BMSCs by Regulating Wnt/ß-Catenin Pathway. Eur. Rev. Med. Pharmacol. Sci. 23 (17), 7232-7246. doi:10.26355/eurrev_201909_18826

Shi, X., Sun, M., Liu, H., Yao, Y., and Song, Y. (2013). Long Non-coding RNAs: a New Frontier in the Study of Human Diseases. Cancer Lett. 339 (2), 159-166. doi:10.1016/j.canlet.2013.06.013

Shi, Y., Parag, S., Patel, R., Lui, A., Murr, M., Cai, J., et al. (2019). Stabilization of lncRNA GAS5 by a Small Molecule and its Implications in Diabetic Adipocytes. Cel Chem. Biol. 26 (3), 319-330. doi:10.1016/j.chembiol.2018.11.012

Skvortsova, K., Iovino, N., and Bogdanović, O. (2018). Functions and Mechanisms of Epigenetic Inheritance in Animals. Nat. Rev. Mol. Cel Biol 19 (12), 774-790. doi:10.1038/s41580-018-0074-2

Steck, E., Boeuf, S., Gabler, J., Werth, N., Schnatzer, P., Diederichs, S., et al. (2012). Regulation of $\mathrm{H} 19$ and its Encoded microRNA-675 in Osteoarthritis and under Anabolic and Catabolic In Vitro Conditions. J. Mol. Med. 90 (10), 1185-1195. doi:10.1007/s00109-012-0895-y

Takahashi, H., Lassmann, T., Murata, M., and Carninci, P. (2012). 5' End-Centered Expression Profiling Using Cap-Analysis Gene Expression and NextGeneration Sequencing. Nat. Protoc. 7 (3), 542-561. doi:10.1038/nprot. 2012.005

Tian, Y., Liu, M., Liu, Y., Shi, C., Wang, Y., Liu, T., et al. (2020). The Performance of 3D Bioscaffolding Based on a Human Periodontal Ligament Stem Cell Printing Technique. J. Biomed. Mater. Res. 109, 1209-1219. doi:10.1002/jbm.a.37114

Toraih, E. A., Alghamdi, S. A., El-Wazir, A., Hosny, M. M., Hussein, M. H., Khashana, M. S., et al. (2018). Dual Biomarkers Long Non-coding RNA GAS5 
and microRNA-34a Co-expression Signature in Common Solid Tumors. PLoS One 13 (10), e0198231. doi:10.1371/journal.pone.0198231

Väänänen, H. K., and Härkönen, P. L. (1996). Estrogen and Bone Metabolism. Maturitas 23 (Suppl. 1), S65-S69. doi:10.1016/0378-5122(96)01015-8

Visconti, V. V., Fittipaldi, S., Ciuffi, S., Marini, F., Isaia, G., D’Amelio, P., et al. (2020). Circulating Long Non-coding RNA GAS5 Is Overexpressed in Serum from Osteoporotic Patients and Is Associated with Increased Risk of Bone Fragility. Ijms 21 (18), 6930. doi:10.3390/ijms21186930

Wang, C., Ke, S., Li, M., Lin, C., Liu, X., and Pan, Q. (2020a). Downregulation of LncRNA GAS5 Promotes Liver Cancer Proliferation and Drug Resistance by Decreasing PTEN Expression. Mol. Genet. Genomics 295 (1), 251-260. doi:10. 1007/s00438-019-01620-5

Wang, Q., Li, Y., Zhang, Y., Ma, L., Lin, L., Meng, J., et al. (2017). LncRNA MEG3 Inhibited Osteogenic Differentiation of Bone Marrow Mesenchymal Stem Cells from Postmenopausal Osteoporosis by Targeting miR-133a-3p. Biomed. Pharmacother. 89, 1178-1186. doi:10.1016/j.biopha.2017.02.090

Wang, X., Zhao, D., Zhu, Y., Dong, Y., and Liu, Y. (2019). Long Non-coding RNA GAS5 Promotes Osteogenic Differentiation of Bone Marrow Mesenchymal Stem Cells by Regulating the miR-135a-5p/FOXO1 Pathway. Mol. Cell Endocrinol. 496, 110534. doi:10.1016/j.mce.2019.110534

Wang, Y., Liu, W., Liu, Y., Cui, J., Zhao, Z., Cao, H., et al. (2018a). Long Noncoding RNA H19 Mediates LCoR to Impact the Osteogenic and Adipogenic Differentiation of mBMSCs in Mice through Sponging miR-188. J. Cel Physiol 233 (9), 7435-7446. doi:10.1002/jcp.26589

Wang, Y., Xiao, X., Zhou, T., Han, D., and Dong, N. (2020b). Novel Mechanisms for Osteogenic Differentiation of Human Aortic Valve Interstitial Cells. J. Thorac. Cardiovasc. Surg. 159 (5), 1742-1753. e1747. doi:10.1016/j.jtcvs. 2019.05.051

Wang, Y., Zeng, X., Wang, N., Zhao, W., Zhang, X., Teng, S., et al. (2018b). Long Noncoding RNA DANCR, Working as a Competitive Endogenous RNA, Promotes ROCK1-Mediated Proliferation and Metastasis via Decoying of miR-335-5p and miR-1972 in Osteosarcoma. Mol. Cancer 17 (1), 89. doi:10. 1186/s12943-018-0837-6

Wei, B., Wei, W., Zhao, B., Guo, X., and Liu, S. (2017). Long Non-coding RNA HOTAIR Inhibits miR-17-5p to Regulate Osteogenic Differentiation and Proliferation in Non-traumatic Osteonecrosis of Femoral Head. PLoS One 12 (2), e0169097. doi:10.1371/journal.pone.0169097

Wu, J., Zhao, J., Sun, L., Pan, Y., Wang, H., and Zhang, W.-B. (2018). Long Noncoding RNA H19 Mediates Mechanical Tension-Induced Osteogenesis of Bone Marrow Mesenchymal Stem Cells via FAK by Sponging miR-138. Bone 108, 62-70. doi:10.1016/j.bone.2017.12.013

Xiao, X., Zhou, T., Guo, S., Guo, C., Zhang, Q., Dong, N., et al. (2017). LncRNA MALAT1 Sponges miR-204 to Promote Osteoblast Differentiation of Human Aortic Valve Interstitial Cells through Up-Regulating Smad4. Int. J. Cardiol. 243, 404-412. doi:10.1016/j.ijcard.2017.05.037

Xie, X., Liu, M., and Meng, Q. (2019). Angelica Polysaccharide Promotes Proliferation and Osteoblast Differentiation of Mesenchymal Stem Cells by Regulation of Long Non-coding RNA H19. Bone Jt. Res. 8 (7), 323-332. doi:10. 1302/2046-3758.87.Bjr-2018-0223.R2

Xue, Y., Ni, T., Jiang, Y., and Li, Y. (2017). Long Noncoding RNA GAS5 Inhibits Tumorigenesis and Enhances Radiosensitivity by Suppressing miR-135b Expression in Non-Small Cell Lung Cancer. Oncol. Res. 25 (8), 1305-1316. doi: $10.3727 / 096504017 \times 14850182723737$

Yan, L., Wu, X., Liu, Y., and Xian, W. (2018). LncRNA Linc00511 Promotes Osteosarcoma Cell Proliferation and Migration through Sponging miR-765. J. Cel Biochem 120, 7248-7256. doi:10.1002/jcb.27999

Yang, L., and Jiang, J. (2019). GAS5 Regulates RECK Expression and Inhibits Invasion Potential of HCC Cells by Sponging miR-135b. Biomed. Res. Int. 2019, 1-11. doi:10.1155/2019/2973289

Yang, Q., Han, Y., Liu, P., Huang, Y., Li, X., Jia, L., et al. (2020). Long Noncoding RNA GAS5 Promotes Osteogenic Differentiation of Human Periodontal Ligament Stem Cells by Regulating GDF5 and P38/JNK Signaling Pathway. Front. Pharmacol. 11, 701. doi:10.3389/fphar.2020.00701

Yang, Q., Jia, L., Li, X., Guo, R., Huang, Y., Zheng, Y., et al. (2018). Long Noncoding RNAs: New Players in the Osteogenic Differentiation of Bone Marrow- and Adipose-Derived Mesenchymal Stem Cells. Stem Cel Rev Rep 14 (3), 297-308. doi:10.1007/s12015-018-9801-5
Yang, X., Yang, J., Lei, P., and Wen, T. (2019). LncRNA MALAT1 Shuttled by Bone Marrow-Derived Mesenchymal Stem Cells-Secreted Exosomes Alleviates Osteoporosis through Mediating microRNA-34c/SATB2 axis. Aging 11 (20), 8777-8791. doi:10.18632/aging.102264

Ye, K., Wang, S., Zhang, H., Han, H., Ma, B., and Nan, W. (2017). Long Noncoding RNA GAS5 Suppresses Cell Growth and Epithelial-Mesenchymal Transition in Osteosarcoma by Regulating the miR-221/ARHI Pathway. J. Cel. Biochem. 118 (12), 4772-4781. doi:10.1002/jcb.26145

Yi, J., Liu, D., and Xiao, J. (2019). LncRNA MALAT1 Sponges miR-30 to Promote Osteoblast Differentiation of Adipose-Derived Mesenchymal Stem Cells by Promotion of Runx2 Expression. Cell Tissue Res 376 (1), 113-121. doi:10.1007/ s00441-018-2963-2

Zambo, I., and Veselý, K. (2014). WHO Classification of Tumours of Soft Tissue and Bone 2013: the Main Changes Compared to the 3rd Edition. Cesk Patol 50 (2), 64-70.

Zhang, J., Zhang, H., Wang, X., Zhao, Y., Fu, Y., and Liu, X. (2020a). PCAT18, as a Novel Differentially Regulated Long Noncoding RNA in Adult Acute Myeloid Leukemia Patients Revealed by Next-generation Sequencing. Int. J. Lab. Hem 42, 858-865. doi:10.1111/ijlh.13305

Zhang, N., Hu, X., He, S., Ding, W., Wang, F., Zhao, Y., et al. (2019a). LncRNA MSC-AS1 Promotes Osteogenic Differentiation and Alleviates Osteoporosis through Sponging microRNA-140-5p to Upregulate BMP2. Biochem. Biophysical Res. Commun. 519 (4), 790-796. doi:10.1016/j.bbrc.2019.09.058

Zhang, X., Du, K., Lou, Z., Ding, K., Zhang, F., Zhu, J., et al. (2019b). The CtBP1Hdac1/2-IRF1 Transcriptional Complex Represses the Expression of the Long Noncoding RNA GAS5 in Human Osteosarcoma Cells. Int. J. Biol. Sci. 15 (7), 1460-1471. doi:10.7150/ijbs.33529

Zhang, Y., Guo, H., Ma, L., Zhu, J., Guo, A., and He, Y. (2020b). Study on Adsorption of microRNA-124 by Long Chain Non-coding RNA MALAT1 Regulates Osteogenic Differentiation of Mesenchymal Stem Cells. Zhongguo Xiu Fu Chong Jian Wai Ke Za Zhi 34 (2), 240-245. doi:10.7507/1002-1892. 201906025

Zhang, Y., Meng, W., and Cui, H. (2018). LncRNA CBR3-AS1 Predicts Unfavorable Prognosis and Promotes Tumorigenesis in Osteosarcoma. Biomed. Pharmacother. 102, 169-174. doi:10.1016/j.biopha.2018.02.081

Zhao, H., Yu, H., Zheng, J., Ning, N., Tang, F., Yang, Y., et al. (2018). Lowlyexpressed lncRNA GAS5 Facilitates Progression of Ovarian Cancer through Targeting miR-196-5p and Thereby Regulating HOXA5. Gynecol. Oncol. 151 (2), 345-355. doi:10.1016/j.ygyno.2018.08.032

Zhao, K., Tu, C., Liang, K., Li, Y., and Yu, Y. (2020a). Long Noncoding RNA MCM3AP Antisense RNA 1 Is Downregulated in Chronic Obstructive Pulmonary Disease and Regulates Human Bronchial Smooth Muscle Cell Proliferation. J. Int. Med. Res. 48 (9), 030006052093521. doi:10.1177/ 0300060520935215

Zhao, L., Zheng, W., and Li, C. (2020b). Association of Long-Chain Non-coding RNA GAS5 Gene Polymorphisms with Prostate Cancer Risk and Prognosis in Chinese Han Population. Medicine (Baltimore) 99 (36), e21790. doi:10.1097/ MD.0000000000021790

Zhao, X., Pathak, J. L., Huang, W., Zhu, C., Li, Y., Guan, H., et al. (2020c). Metformin Enhances Osteogenic Differentiation of Stem Cells from Human Exfoliated Deciduous Teeth through AMPK Pathway. J. Tissue Eng. Regen. Med. 14, 1869-1879. doi:10.1002/term.3142

Zheng, S., Jiang, F., Ge, D., Tang, J., Chen, H., Yang, J., et al. (2019). LncRNA SNHG3/miRNA-151a-3p/RAB22A axis Regulates Invasion and Migration of Osteosarcoma. Biomed. Pharmacother. 112, 108695. doi:10.1016/j.biopha.2019. 108695

Zheng, S., Li, M., Miao, K., and Xu, H. (2020). IncRNA GAS5-promoted Apoptosis in Triple-negative Breast Cancer by Targeting miR-378a-5p/SUFU Signaling. J. Cel Biochem 121 (3), 2225-2235. doi:10.1002/jcb.29445

Zhou, B., Yang, H., Yang, C., Bao, Y.-1., Yang, S.-m., Liu, J., et al. (2021). Translation of Noncoding RNAs and Cancer. Cancer Lett. 497, 89-99. doi:10.1016/j.canlet. 2020.10.002

Zhou, P., Li, Y., Di, R., Yang, Y., Meng, S., Song, F., et al. (2019). H19 and Foxc2 Synergistically Promotes Osteogenic Differentiation of BMSCs via Wnt- $\beta$ catenin Pathway. J. Cel Physiol 234 (8), 13799-13806. doi:10.1002/jcp.28060

Zhou, Q. P., Zhang, F., Zhang, J., and Ma, D. (2018a). H19 Promotes the Proliferation of Osteocytes by Inhibiting P53 during Fracture Healing. Eur. 
Rev. Med. Pharmacol. Sci. 22 (8), 2226-2232. doi:10.26355/ eurrev_201804_14808

Zhou, S., Yu, L., Xiong, M., and Dai, G. (2018b). LncRNA SNHG12 Promotes Tumorigenesis and Metastasis in Osteosarcoma by Upregulating Notch2 by Sponging miR-195-5p. Biochem. Biophysical Res. Commun. 495 (2), 1822-1832. doi:10.1016/j.bbrc.2017.12.047

Zong, Y., Zhang, Y., Sun, X., Xu, T., Cheng, X., and Qin, Y. (2019). miR-221/222 Promote Tumor Growth and Suppress Apoptosis by Targeting lncRNA GAS5 in Breast Cancer. Biosci. Rep. 39 (1), BSR20181859. doi:10.1042/bsr20181859

Conflict of Interest: The authors declare that the research was conducted in the absence of any commercial or financial relationships that could be construed as a potential conflict of interest.
Publisher's Note: All claims expressed in this article are solely those of the authors and do not necessarily represent those of their affiliated organizations, or those of the publisher, the editors, and the reviewers. Any product that may be evaluated in this article, or claim that may be made by its manufacturer, is not guaranteed or endorsed by the publisher.

Copyright $\odot 2022$ Zhou, Chen, Huang, Liu, Chen and Qin. This is an open-access article distributed under the terms of the Creative Commons Attribution License (CC $B Y)$. The use, distribution or reproduction in other forums is permitted, provided the original author(s) and the copyright owner(s) are credited and that the original publication in this journal is cited, in accordance with accepted academic practice. No use, distribution or reproduction is permitted which does not comply with these terms. 